\title{
EDUCAÇÃO EM SAÚDE E QUALIDADE DE VIDA PARA O COMBATE DA HIPERTENSÃO ARTERIAL SISTÊMICA EM UMA UNIDADE COMERCIAL DE SALVADOR, BA
}

\author{
Gleide Regina de Sousa Almeida Oliveira ${ }^{1}$ \\ Luiz Cezar Mota Ramos ${ }^{2}$ \\ Marcelo Santana de Bastos Melo²
}

\begin{abstract}
RESUMO: Este trabalho trata-se de um relato de experiência elaborado a partir do projeto de extensão "Educação em Saúde e Qualidade de Vida Para a Comunidade", que busca transmitir conhecimentos e informações sobre promoção e prevenção à saúde para a comunidade por meio da troca de experiências e prática de ações no âmbito educacional, gerando um elo construtivo entre a universidade e a comunidade. A abordagem do relato de experiência relaciona-se a uma atividade específica realizada em uma unidade comercial de grande porte da comunidade adjacente ao centro universitário vinculado ao projeto de extensão no qual foi trabalhada a Hipertensão Arterial Sistêmica (HAS) entre os funcionários. Realizaram-se atividades com materiais didáticos sobre a HAS e aferição da pressão arterial dos funcionários. Avaliados os resultados, 15\% apresentou a pressão arterial (PA) elevada (maior ou igual a 140X90 mm Hg), enquanto o restante dos funcionários apresentou níveis pressóricos dentro dos parâmetros desejáveis. Transmitiramse, deste modo, as devidas orientações de como manter hábitos adequados para o controle ou prevenção da HAS. Assim, é perceptível o quão importante é a construção dessas atividades em parceria com as empresas, visando à melhoria da qualidade de vida dos trabalhadores.
\end{abstract}

PALAVRAS-CHAVE: Hipertensão. Enfermagem. Educação em saúde.

Health education and quality of life for the battle of systemic hypertension in a business unit of Salvador, $B A$

\begin{abstract}
This work is a report of the experience drawn from the Extension Project "Education in Health and Quality of Life for the Community" which seeks to impart knowledge and information on health promotion and prevention to the community through the exchange of experiences and practical actions in the educational generating a constructive link between the university and the community. The approach of the experience report relates to a specific activity performed in the large commercial community adjacent to the university center linked to the extension project, which was crafted Hypertension (HBP) among employees. There were activities with educational material on hypertension and besides and blood pressure measurement of the employees. The results evaluated, $15 \%$ had blood pressure (BP) (greater than or equal to $140 \mathrm{X} 90 \mathrm{~mm} \mathrm{Hg}$ ) while the remainder of employees showed blood pressure within desirable parameters. Transmitted is thus appropriate guidance on how to maintain habits suitable for the control or prevention of hypertension. Thus, it is apparent how important is the construction of these activities in partnership with companies aiming to improve the quality of life of workers.

\footnotetext{
${ }^{1}$ Mestre em Enfermagem pela Universidade Federal da Bahia, professora assistente no curso de Enfermagem do Centro Jorge Amado, coordenadora do projeto de extensão "Educação em Saúde e Qualidade de Vida para a Comunidade" (gleideenf@gmail.com).

2 Acadêmicos do Curso de Enfermagem do Centro Universitário Jorge Amado, participante do projeto de extensão "Educação em Saúde e Qualidade de Vida para a Comunidade" (luizinho_lcmr@hotmail.com; celu_melu@hotmail.com). 
KEYWORDS: Hypertension. Nursing. Health education.

\section{INTRODUÇÃO}

O projeto de extensão "Educação em Saúde e Qualidade de Vida Para a Comunidade" é composto por 15 acadêmicos do Curso de Bacharelado em Enfermagem do Centro Universitário Jorge Amado, cursando entre o $6^{\circ}$ e o $8^{\circ}$ período letivo, dentre os quais um docente assume papel de orientador, sendo responsável pelas atividades a serem desenvolvidas.

De acordo com Chobanian e colaboradores (2003), a hipertensão é definida como uma pressão arterial sistólica superior a $140 \mathrm{~mm} \mathrm{Hg} \mathrm{e} \mathrm{uma} \mathrm{pressão} \mathrm{diastólica} \mathrm{superior} \mathrm{a} 90 \mathrm{~mm} \mathrm{Hg}$, na média de duas ou mais medições exatas da pressão arterial, obtidas durante dois ou mais contatos com o profissional de saúde.

A pressão arterial alta, conhecida como Hipertensão Arterial Sistêmica, pode resultar de uma alteração no débito cardíaco, uma modificação da resistência periférica ou de ambas (SMELTZER et al., 2009). Assim, sabe-se que, no Brasil, dentre as causas de óbitos conhecidas, 33\% envolvem as doenças cardiovasculares. Entre os anos 1996 e 1999, no setor público, essa doença foi considerada como a primeira causa de hospitalização no período e correspondeu a $17 \%$ das internações de pessoas com idade entre 40 e 59 anos e $29 \%$ daquelas com 60 ou mais anos (BARRETO et al., 2000 apud PASSOS; ASSIS; BARRETO, 2006).

Segundo Lessa (2010), a Hipertensão Arterial Sistêmica (HAS) é a enfermidade vascular mais frequente no mundo, sendo o fator de maior risco para doenças cerebrovasculares, com causa predominante de morte no Brasil. Dessa forma, a Hipertensão Arterial Sistêmica (HAS) é uma doença crônica não transmissível, silenciosa, de alta prevalência, cujo diagnóstico e controle são imprescindíveis no manejo de graves moléstias, como insuficiência cardíaca congestiva, doenças cerebrovasculares, infarto agudo do miocárdio, nefropatia hipertensiva, insuficiência vascular periférica e retinopatia hipertensiva (RABETTI; FREITAS, 2011).

A hipertensão arterial é responsável por 25\% da etiologia multifatorial da cardiopatia isquêmica e por $40 \%$ dos acidentes vasculares cerebrais, além de considerada um importante fator de risco para doenças decorrentes de aterosclerose e trombose que se enfatizam, predominantemente, por acometimento cardíaco, cerebral, renal e vascular periférico. Devido a esses agravos, a HAS é considerada como origem das doenças cardiovasculares e, portanto, caracteriza-se como um dos fatores para a redução da qualidade e longevidade de vida (PASSOS; ASSIS; BARRETO, 2006).

Para Moreira, Moraes e Luiz (2011), a HAS é uma enfermidade, geralmente, desconhecida e que exige uma complexidade de recursos necessários para seu controle, como serviços médicos, pessoal treinado e medicamentos. Apenas metade dos portadores dessa doença recebe algum tipo de assistência médica para seu controle.

A presença da hipertensão pode ter uma influência muito negativa na jornada de trabalho dos empregados a partir do momento que está relacionada com dificuldades ao adormecer, sono interrompido e cansaço ao acordar. Desta forma, o trabalhador irrita-se com frequência e, consequentemente, relata sensações de mal-estar, acarretando baixo rendimento (PAVAN et al., 2005). 
Os profissionais de saúde têm como objetivo realizar a prevenção e a promoção da saúde do trabalhador, para que, assim, o funcionário saiba de sua real situação de vida. Assim, empresas e unidades comerciais devem priorizar os serviços de saúde prestados por profissionais adequados, principalmente enfermeiros ${ }^{3}$, a fim de orientar e educar os trabalhadores para a saúde.

Em verdade, todas as empresas de grande porte deveriam aderir a essa assistência, fazendo constar, no quadro do Setor de Medicina do Trabalho, uma equipe multiprofissional (médico, enfermeiro, dentista, psicólogo e nutricionista) e exigindo exames periódicos e de rotina, a fim de facilitar a qualidade de vida dos funcionários da instituição.

Caso a HAS seja diagnosticada antecipadamente, a mudança do estilo de vida é considerada fator crucial para a redução dos níveis pressóricos da pressão arterial. Algumas ações na mudança de hábitos podem ser aderidas como opção para uma melhor qualidade da saúde humana, como atividades físicas regulares, redução do tabagismo e dieta alimentar saudável (BATTAGIN et al., 2010).

O projeto "Educação em Saúde e Qualidade de Vida Para a Comunidade" teve como um dos principais objetivos disseminar informações pertinentes à área de saúde para a comunidade, tornando a percepção do público envolvido sobre prevenção e tratamento da HAS mais acessível. Isto porque a educação em saúde é um processo no qual ocorre uma troca de experiências entre os saberes do profissional e as vivências do dia-a-dia da comunidade.

A partir da construção de um vínculo entre os funcionários da unidade comercial participante e os integrantes do projeto, houve facilidade deles relatarem suas experiências e dúvidas, norteando as orientações mais adequadas para cada um. Assim, o ambiente da empresa tornou-se um local mais favorável para o desenvolvimento da educação em saúde de seus trabalhadores.

O projeto trabalha, portanto, temáticas pertinentes à saúde na comunidade, criando um vínculo entre a educação e a promoção da saúde para um bem-estar geral. Neste estudo, ressalta-se a relevância da HAS como um dos principais fatores de risco para a vida dos trabalhadores. Dessa forma, foi fomentada a ideia do que se trata a doença, como preveni-la e como conviver com tal agravo a saúde.

\section{METODOLOGIA}

Foi desenvolvida, por meio do projeto de extensão, uma ação em saúde localizada em uma unidade comercial de Salvador/BA, no período de 21 a 27 de outubro de 2011, com funcionários de diversos setores de uma empresa de material de construção durante o intervalo do almoço. O grupo de quinze componentes dividiu-se em subgrupos, os quais abordaram assuntos diferentes: doenças cardiovasculares, Diabetes Mellitus (DM), câncer de mama e de próstata, tabagismo, etilismo e obesidade. As atividades foram desenvolvidas por meio de palestras, exposições de maquetes, cartazes, panfletos, ilustrações, dinâmicas em grupo, aferição da pressão arterial, coleta de dados para a realização do Índice de Massa Corpórea (IMC) e a medida da circunferência abdominal.

\footnotetext{
${ }^{3} \mathrm{O}$ enfermeiro atua como um agente do cuidar da saúde do trabalhador, estabelecendo uma relação social, assumindo seu compromisso ético e profissional. 
De forma geral, as discussões iniciaram-se a partir das explicações acerca do caráter fisiológico e suas alterações, causas, consequências, riscos e prevenção de cada doença abordada. Dentre as enfermidades cardiovasculares trabalhadas no projeto, a mais discutida foi a HAS, devido à noção de sua existência ser prevalente e questionada pelos funcionários da empresa, apesar de o conhecimento não representar entendimento sobre a morbidade e nem atitude.

Um dos subgrupos ficou responsável pela aferição da pressão arterial, pelo cálculo do IMC, pela medição da circunferência abdominal e explicação sobre valores considerados normais de cada medida, além de informar quais ações em saúde os funcionários deveriam realizar para ter uma melhor qualidade de vida e hábitos saudáveis. A medida do IMC, que envolve os valores do peso e da altura, foi realizada por dois componentes da equipe em um dos subgrupos e seu cálculo foi realizado de acordo com a fórmula IMC $=[$ peso $(\mathrm{kg})] /[\text { altura }(\mathrm{m})]^{2}$.

A partir de então, os discentes envolvidos no projeto explicavam para cada funcionário sobre os valores considerados normais para a Organização Mundial de Saúde (OMS) e eles recebiam as devidas orientações sobre quantos quilos deveriam adquirir ou perder para representar um padrão de índice de massa corpórea desejável. Consideramos aqueles com a classificação do IMC $<25$ como normais/eutróficos; os que apresentavam o IMC entre 25,0 e 29,9 foram categorizados com sobrepeso; e aqueles com IMC $\geq 30,0$, classificados como obesos (WORLD HEALTH ORGANIZATION, 1998 apud AMER; MARCON; SANTANA, 2011). Além disso, foram dadas as devidas explicações acerca de hábitos alimentares saudáveis e práticas de atividades físicas diárias, considerando como regular a prática de exercícios físicos, no mínimo três vezes na semana, durante 30 minutos por dia.

O IMC inadequado está intimamente ligado a fatores condicionantes de doenças cardiovasculares, como um maior índice de infarto agudo do miocárdio, além de aumento da pressão arterial devido à elevação da concentração de tecido adiposo, estando esses fatores diretamente relacionados à medida da circunferência abdominal.

A coleta dos dados da circunferência abdominal foi realizada por um dos componentes do subgrupo, por meio de uma fita métrica colocada na inserção da cicatriz abdominal, circundando o abdômen do examinado. Foi exibida uma tabela dividida por sexo (masculino e feminino) com as suas respectivas medidas consideradas aceitáveis e, de acordo com a medida de cada um, foram repassadas as devidas orientações.

O subgrupo encarregado por tais atividades ainda ficou responsável por organizar uma tabela que registrasse todos os dados supracitados, além da medida da pressão arterial dos examinados. Por meio do uso estetoscópio e do tensiômetro, foram encontrados os valores em milímetros de mercúrio $(\mathrm{mm} \mathrm{Hg})$ da pressão arterial dos funcionários.

Foram explicados os valores considerados ideais de acordo com a classificação do JNC 71, a qual considera quatro níveis em mmHg: normal (PA sistólica $<120$ e PA diastólica $<80$ ); préhipertensão (PA sistólica entre 120-139 ou PA diastólica entre 80-89); hipertensão estágio 1 (PA sistólica entre 140-159 ou PA diastólica entre 90-99) e hipertensão estágio 2 (PA sistólica $\geq 160$ ou PA diastólica $\geq 100$ ), para se ter uma PA adequada, e a partir de então passadas as explicações 
sobre o assunto a depender dos valores da PA encontrados (CHOBANIAN et al., 2003).

\section{RESULTADOS E DISCUSSÃO}

A contribuição do projeto de extensão na unidade comercial partiu da necessidade de se abordar questões sobre a saúde no ambiente de trabalho, focando a promoção da qualidade de vida do trabalhador. Por meio da relação saúde e ambiente de trabalho é possível construir novos conhecimentos em torno dos agravos provocados pela mecânica do trabalho e maus hábitos de vida, a fim de evitar e reduzir consequências negativas para o bem estar do funcionário.

A origem de algumas doenças surge a partir de fatores socioculturais, biológicos e ambientais, para as quais o tratamento medicamentoso nem sempre é a única solução. A prática da ação em saúde é uma porta de entrada para novos horizontes no que diz respeito a uma outra maneira paliativa na contribuição da saúde da sociedade.

O projeto de extensão foi bem aceito pela empresa e pelos funcionários, sendo perceptível a carência de informações sobre assuntos pertinentes a saúde geral dos indivíduos. Ocorreu uma participação ativa dos funcionários, notada a partir do interesse nas atividades envolvidas, levantando um diálogo dinâmico e empático.

No decorrer das tarefas do subgrupo destinado a aferição da pressão arterial, verificou-se que foram atendidos setenta funcionários, em média, dentre os quais, aproximadamente, 15\% a apresentou elevada (maior que $140 \mathrm{X} 90 \mathrm{~mm} \mathrm{Hg}$ ), enquanto que o restante apresentou níveis pressóricos dentro dos parâmetros desejáveis.

Diante das circunstâncias, foram discutidas as possíveis causas da HAS e levantadas questões sobre hábitos alimentares, sedentarismo, tabagismo e etilismo - fatores de risco que contribuem para o agravamento da elevação da pressão arterial. Alguns funcionários, após aferição da pressão arterial, relataram em suas falas o alto consumo de sal, como, por exemplo, a fala de um trabalhador após aferição da pressão arterial em 160X80 mm Hg: "Foi porque eu acabei de almoçar e exagerei no sal de cozinha". Outro, cuja pressão arterial estava 140X90 mm Hg, relatou: "Toda vez que eu me esqueço de tomar meu anti-hipertensivo acontece isso".

A mudança no estilo de vida é uma tarefa árdua e interligada à resistência das pessoas, uma vez que não conseguem abrir mão de seus maus hábitos. A educação em saúde é importante para direcionar as pessoas para estas mudanças, visando a prevenção e o controle dos fatores de riscos da HAS e o cultivo de hábitos e atitudes saudáveis, possibilitando-lhes a melhora de sua saúde, de forma geral, e seu bem-estar físico e mental (SANTOS; LIMA, 2008).

No desenvolvimento das atividades, foi perceptível que os trabalhadores não estavam recebendo a assistência de saúde adequada em seu local de trabalho. Observou-se que o IMC de alguns funcionários estava elevado, chegando a indicar obesidade de grau 1 e 2, diretamente relacionada com a elevação da pressão arterial. Sendo assim, foi aconselhada a prática de exercícios físicos 
e uma dieta mais balanceada, com baixos índices de gordura e cloreto de sódio, associada a um maior consumo de frutas e hortaliças. Neste contexto, uma alimentação baseada em alimentos industrializados, rica em gordura e sal pode ser fator predisponente para agravos à saúde, uma vez que mantêm um nível consideravelmente alto de sódio e potássio, estando intimamente associado a níveis pressóricos elevados (MOLINA et al., 2003).

Outro fator detectado que possui relação com a elevação da pressão arterial foi a circunferência abdominal (CA). De acordo com os resultados encontrados, foram repassadas orientações semelhantes ao IMC e à HAS. Foram encontrados valores maiores que $112 \mathrm{~cm}$ de circunferência abdominal em homens e, em mulheres, valores maiores que $90 \mathrm{~cm}$. Rezende e colaboradores (2006) ressaltam que, em mulheres, o grau de risco para doenças cardiovasculares é aumentado quando $\mathrm{CA}>80 \mathrm{~cm}$ e, em homens, quando $\mathrm{CA}>94 \mathrm{~cm}$; e risco muito aumentado quando $\mathrm{CA}$ $>88 \mathrm{~cm}$ (mulheres) $\mathrm{CA}>102 \mathrm{~cm}$ (homens).

Após os esclarecimentos, percebeu-se, por parte dos funcionários, certo estímulo para adotar melhorias em seus hábitos de vida. Associada à redução da pressão arterial e da obesidade, a atividade desenvolvida perpassou por outros temas como: tolerância à glicose, doenças degenerativas articulares, apneia do sono e sintomas depressivos.

As orientações em saúde repassadas podem contribuir para a diminuição da resistência a atividades físicas e ao aumento da autoestima das pessoas, significando, ao final, em uma melhora da qualidade de vida (BARRETO-FILHO; CONSOLIM-COLOMBO; LOPES, 2002).

Desta forma, acredita-se que projetos de extensão como o relatado tornam-se instrumentos importantes para a diminuição de comorbidades e doenças crônicas, estimulando a qualidade de vida da comunidade extra-acadêmica.

\section{CONSIDERAÇÕES FINAIS}

A partir do trabalho desenvolvido, foi possível confirmar a importância de se trabalhar a educação em saúde na comunidade.

Notou-se que o resultado final foi construtivo tanto para os acadêmicos de Enfermagem, quanto para os funcionários que participaram do projeto. As atividades desenvolvidas contribuíram para a formação de um senso comum sobre a HAS e outras patologias, evidenciando uma assimilação e uma capacitação do trabalhador em relação aos cuidados adequados com sua saúde.

Percebeu-se a relevância de se desenvolver mais trabalhos relacionados ao tema proposto, posto que, uma vez incentivado e incrementado o desenvolvimento de autonomia para prevenção de agravos da saúde e sua promoção, as pessoas irão desempenhar um papel de disseminadores do conhecimento, aumentando a perspectiva de vida da população e seu bem- estar físico e mental. 


\section{REFERÊNCIAS}

AMER, N. M.; MARCON, S. S.; SANTANA, R. G. Índice de massa corporal e hipertensão arterial em indivíduos adultos no centro-oeste do Brasil. Arquivos Brasileiros de Cardiologia, Rio de Janeiro, v. 96, n.1, p. 47-53, 2011.

BARRETO-FILHO, J. A. S.; CONSOLIM-COLOMBO, F. M.; LOPES, H. F. Hipertensão arterial e obesidade: causa secundária ou sinais independentes da síndrome plurimetabólica? Rev Bras Hipertens., São Paulo, v. 9, p. 174-184, abr.-jun. 2002.

BATTAGIN, A. M. et al. Resposta pressórica após exercício resistido de diferentes segmentos corporais em hipertensos. Arquivos Brasileiros de Cardiologia, São Paulo, v.95, n.3, set. 2010. Disponível em: $<$ http://www.scielo.br/scielo.php?pid=S0066-782X2010001300018\&script=sci_ arttext>. Acesso em: 24 mar. 2012

CHOBANIAN A.V. et al. The seventh report of the joint national committee on prevention, detection, evaluation and treatment of high blood pressure. Hypertension, v. 42, p. 1206-1252, dez. 2003.

COLOMBO, F. M.; LOPES, H. F. Hipertensão arterial e obesidade: causa secundária ou sinais independentes da síndrome plurimetabólica? Rev. Bras. Hipertens., São Paulo, v. 9, p. 174184, abr.-jun. 2002.

LESSA, I. Hipertensão arterial sistêmica no Brasil: tendência temporal. Cad. Saúde Pública, Rio de Janeiro, v. 26, n. 8, ago. 2010. Disponível em: $<$ http://www.scielo.br/scielo.php?script=sci_ar ttext\&nrm=iso\&lng=pt\&tlng=pt\&pid=S0102-311X2010000800001>. Acesso em: 14 mar. $20 \overline{1} 2$.

MOLINA, M. C. B. et al. Hipertensão arterial e consumo de sal em população urbana. Rev Saúde Pública, São Paulo, v. 37, n. 6, p. 743-50, 2003.

MOREIRA,J. P. L.; MORAES, J. R.; LUIZ, R. R. Utilização de consulta médica e hipertensão arterial sistêmica nas áreas urbanas e rurais do Brasil, segundo dados da PNAD 2008. Ciênc. Saúde Coletiva, Rio de Janeiro, v. 16, n. 9, set. 2011. Disponível em: < http://www.scielo.br/ scielo.php?script=sci_arttext\&nrm=iso\&lng=pt\&tlng=pt\&pid=S1413-81232011001000014>. Acesso em: 14 mar. 2012.

PASSOS, V. M. A.; ASSIS, T. D.; BARRETO, S. M. Hipertensão arterial no Brasil: estimativa de prevalência a partir de estudos de base populacional. Epidemiol. Serv. Saúde, Brasília, v. 15, n. 1, mar. 2006. Disponível em: <http://scielo.iec.pa.gov.br/scielo.php?script=sci_ arttext\&pid=S1679-49742006000100003\&lng=pt\&nrm=iss\&tlng=pt $>$. Acesso em: 13 mar. 2012.

PAVAN, R. M. S. et al. Diagnósticos de enfermagem em trabalhadores hipertensos de uma empresa de transporte urbano coletivo. Revista Eletrônica de Enfermagem, Goiânia, v. 7, n. 2, p. 173-178, 2005.

RABETTI, A. C.; FREITAS, S. F. T. Avaliação das ações em hipertensão arterial sistêmica na atenção básica. Rev. Saúde Pública, São Paulo, v. 45, n. 2, abr. 2011. Disponível em: <http:// www.scielosp.org/scielo.php?script=sci_arttext\&pid=S0034-89102011000200004\&lang=pt\&t $\operatorname{lng}=\mathrm{pt}>$. Acesso em: 21 mar. 2012. 
REZENDE, F. A. C. et al,. Índice de Massa Corporal e Circunferência Abdominal: Associação com Fatores de Risco Cardiovascular. Arquivos Brasileiros de Cardiologia, São Paulo, v. 87, n. 6, p. 728-734, dez. 2006.

SANTOS, Z. M. S. A.; LIMA, H. P. Tecnologia educativa em saúde na prevenção da hipertensão arterial em trabalhadores: análise das mudanças no estilo de vida. Texto Contexto Enferm., Florianópolis, v.17, n. 1, p. 90-7, jan.-mar. 2008.

SMELTZER, S. C. et al. Tratado de enfermagem médico-cirúrgica. Rio de Janeiro: Guanabara Koogan, 2009.

Submetido em 19 de abril de 2012.

Aprovado em 17 de julho de 2012. 